Fig. 2. Aus dem kleineren Tumor desselben Falles. Ein Lymphgefäss des Bindegewebes von amyloider Substanz ausgefüllt; an beiden Längsseiten bandartige Riesenzellen; an dem einen Pol eine Riesenzelle, deren Fortsätze in Spalten des Blockes hineinwachsen.

Fig. 3. Aus dem Tumor des Falles Kolber. Verzweigtes Knochenbälkchen, zum Theil von Bindegewebe, zum Theil von amyloider Substanz begrenzt. Nach rechts von ihm im Bindegewebe Fettzellen und eine Gruppe von Schleimdrüsenbläschen, deren einige verdickte und amyIoide Membranae propriae besitzen.

Fig. 4. Ebendaher. Amyloide Blöcke im Lumen von Lymphgefässen. Auf der bindegewebigen Wand theils glatte Endothelien, theils vielkernige Riesenzellen. Nach links rom grössten der Kanäle ein amyloider Balken im Bindegewebe mit angelagerter Riesenzelle.

\title{
XV.
}

\section{Ueber die Einwirkung barometrisch verschiedener Luftarten auf den intrapleuralen und den Blut-Druck bei Kaninchen.}

(Aus dem Pneumatischen Institut des Krankenhauses der jüdischen Gemeinde in Berlin.)

Von Dr. E. Aron, Assistenzarzt.

Innerhalb des Pleuraraumes herrscht bekanntermaassen vom ersten Athemzuge an ein negativer Druck in Folge des Zuges, welchen die über ihr natürliches Volum ausgedehnten Lungen ausüben.

Dieser intrapleurale Druck, mit dem wir uns im Folgenden unter gewissen Bedingungen zu beschäftigen haben werden, ist negativ, wird stärker negativ bei der Inspiration, weniger negativ mit der Exspiration. Je tiefer eingeathmet wird, ein um so grösserer negativer Druck wird in der Pleurahöhle herrschen müssen; je stärker exspirirt wird, um so mehr nähert sich der intrapleurale Druck der Nulllinie, ja kann schliesslich bei forcirter Ausathmung diese sogar kreuzen, d. h. positiv werden. Wir werden daher jeder Zeit aus der Grösse des intrapleuralen 
Druckes in der Lage, uns über die Lungenstellung ein Bild zu machen, und berechtigt sein, uns dann auch über den Stand des Zwerchfells in der betreffenden Athmungsphase zu orientiren. Jedenfalls dürfte das unbestreitbar sein: je grösser der negative, intrapleurale Druck, um so tiefer die Inspirationsstellung der Lunge und des Thorax im Allgemeinen, um so tiefer steht das $\mathrm{Zwerchfell}$ in diesem Momente der Respiration, und umgekehrt: je kleiner der intrapleurale, negative Druck, um so mehr entspricht dies der Exspirationsstellung des Thorax, um so böher steht das Diaphragma. Diese Verhältnisse sind so klar und durchsichtig, dass es fast so scheinen könnte, als wenn es überflüssig wäre, darüber ein Wort zu verlieren. Ich habe dieselben trotzdem ausführen zu müssen geglaubt, da wir sie bei unseren Untersuchungen als Basis benutzen, auf der wir bauen.

In Folgendem habe ich eine Reihe von Versuchen angestellt, um den Einfluss von einerseits comprimirter und andererseits verdünnter Luft auf den intrapleuralen Druck bei einer Anzahl von Kaninchen zu studiren. Bei diesen Experimenten verfuhr ich folgendermaassen:

Die Thiere wurden in bekannter Weise auf einem Kaninchenbrett festgebunden (von einer Narkotisirung derselben wurde vollkommen Abstand genommen), und dann wurde eine Canüle mit konischer Spitze, welche dicht hinter dieser einige kleine seitliche Fenster besitzt, in einen Intercostalraum eingestossen. Die Canüle wird mittelst eines Gummischlauches mit einem Glycerinmanometer luftdicht verbunden. Der freie Schenkel des Manometers ist mit einem Schwimmer und Zeichenfeder armirt, und diese Feder verzeichnet auf einer Kymographiontrommel die Druckschwankungen des den Pleuradruck angebenden Manometers.

Es ist ein leichtes, mittelst einer nicht all' zu scharf zugespitzten Canüle in den Pleuraraum des Versuchsthieres zu gelangen, ohne die Lunge desselben zu verletzten. Das Manometer zeigt in promptester Weise an, wann wir in den Pleuraraum eingedrungen sind, indem es einen negativen Druck und von diesem Momente an die Schwankungen angiebt, welche der intrapleurale Druck bei der Athmung erleidet.

Es war dann nothwendig, die Canüle in der richtigen 
Stellung unverrückt im Thorax zu fixiren, ohne jedoch die Respiration des Thieres zu erschweren. Zu dem $Z$ wecke hatte ich die betreffende Canüle, senkrecht zu ihr, durch ein Stückchen Gummischlauch gesteckt, welcher in seiner Wand mit zwei kleinen, sich gegenüber befindlichen Löchern versehen war. Wurde die Canüle durch diese Löcher hindurchgeschoben, so wurde sie in Folge der Elasticität des Schlauches mit Leichtigkeit in jeder Lage von selbst festgehalten. Dann zogen wir, nachdem die so armirte Canüle in den Pleuraraum eingestossen war, durch die Brusthaut des Thieres dicht an der Einstichstelle der Canüle einen Faden zu beiden Seiten des kleinen Stückchens Gummischlauch und banden nun über diesen selbst zu. Es war auf diese Weise ein leichtes einerseits die Canüle überhaupt und andererseits so zu befestigen, dass sie unverrückbar der Athmung folgen konnte, ohne diese selbst irgendwie zu behindern.

Gleichzeitig mit diesen Versuchen stellten wir noch Blutdruckbestimmungen an, indem eine Glascanüle dem Kaninchen in eine Carotis eingebunden wurde. Diese Canüle stand mit einem Quecksilbermanometer, welches mit Schwimmer und Schreibfeder ausgestattet war, in Verbindung. Wir bestimmten also gleichzeitig mit dem intrapleuralen Druck bei unseren Experimenten noch den Blutdruck und konnten also bei unseren Versuchen im pneumatischen Cabinet gleichzeitig Aenderungen einerseits des Blutdruckes und andererseits des intrapleuralen Druckes unter dem Einflusse des veränderten umgebenden Luftdruckes zum Gegenstand des Studiums machen. Da der Blutdruck in so hohem Maasse von dem intrapleuralen Drucke tangirt wird, wie dies ja zur Genüge bekannt ist, musste es von einigem Interesse sein, gleichzeitig beides zu beobachten. Die erhaltenen Curven und die beigegebenen Tabellen geben in übersichtlicher und ausreichender Weise Aufschluss über Druckänderungen in beiden Systemen, dem Respirations- und dem Circulationssystem, unter verändertem äusseren Luftdruck.

Waldenburg') sagt: "Eine andere rein mechanische Wirkung der pneumatischen Cabinette auf die Respiration als das

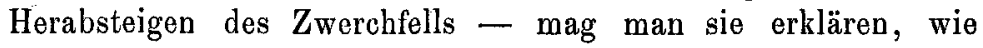

1) Die pneumatische Behandlung der Respirations- und Circulationskrankheiten. Berlin 1880. S. 569. 
man wolle - ist bisher überhaupt nicht nachgewiesen". In Folgendem dürfte es nun wohl gelingen, in anderer Weise, an der Hand des intrapleuralen Druckes, den Beweis zu erbringen, dass und in welchem Sinne comprimirte and verdünnte Luft auf die Mechanik der Athmung einwirkt.

Man hat diesen Befund des Herabsteigens des Zwerchfells in comprimirter Luft zu erklären versucht; in verdünnter Luft wird es sich im Gegentheil um ein Heraufsteigen des $Z$ werchfells handeln müssen. v. Vivenot und Panum sehen in der Compression der Darmgase bei Aufenthalt in verdichteter Luft die Haupt-, Paul Bert sogar die einzige Ursache für das Tiefertreten des $Z$ werchfells. Ich bin weit davon entfernt, bestreiten $z \mathfrak{u}$ wollen, dass dieser Umstand in der That eine wichtige Rolle spielen muss, glaube jedoch nicht verschweigen zu dürfen; dass er allein nach meinem Dafürhalten nicht völlig zur Erklärung des mechanischen Effektes comprimirter Luft auf den Thierkörper ausreicht.

Bekanntlich besitzen Kaninchen und andere herbivore Thiere keine oder nur sehr wenig Darmgase, und diese wenigen Darmgase dürften denn doch wohl kaum ausreichen, um den hohen Grad der Beeinflussung der Lungen- und $Z$ werchfellstellung, wie wir ihn zu verzeichnen haben werden, in befriedigender Weise plausibel zu machen. Waldenburg's ${ }^{1}$ ) Versuch, diese mechanische Beeinflussung comprimirter Luft auf die Lunge zu erklären, mag daher hier Erwähnung finden: „Beim Aufenthalt in comprimirter Luft findet zwar der gleiche Druck auf die innere Oberfläche der Lunge, wie auf den Thorax statt; es kann deshalb zwar der Thorax nicht ausgedehnt werden, aber dennoch kann die Lunge als ein elastisches Gewebe dem Drucke mehr nachgeben, als die weniger elastische Thoraxwand. Am wenigsten nun dem äusseren Drucke ausgesetzt ist das $Z$ werchfell, weil es, durch die Bauchhöhle geschütat, nicht direct von dem auf der äusseren Körperoberfläche lastenden Gegendruck betroffen wird, oder mit anderen Worten durch die Widerstände der dazwischen liegenden Körperpartien von einem Theile des Druckes entlastet wird". $O b$ dieser Erklärungsversuch Waldenburg's in der That zutrifft, will ich nicht entscheiden; jedenfalls eri) a. a. O. S. 568 . 
scheint or nicht ganz irrationell und mangels eines besseren wohl annehmbar.

Exacte Untersuchungen, welche die Wirkungen comprimirter und auch verdünnter Luft auf den Mechanismus der Respirationsorgane betreffen, sind noch immer nicht eben zahlreich und oft nicht so eindeutig, dass fernere Untersuchungen auf diesem Gebiete überfiüssig erscheinen könnten.

Am bekanntesten von dieser Art Experimente sind zunächst jene von J. Lange am Pneumatometer, durch welche constatirt wurde, dass der negative Inspirations- und positive Exspirationsdruek in comprimirter Luft wächst. v. Vivenot und Stembo ${ }^{1}$ ) haben dann eine Steigerung der vitalen Capacität im pneumatischen Cabinet in verdichteter Luft nachgewiesen, Schyrmunski2) eine Abnahme derselben in verdünnter Luft. Bliden hat ferner in seiner beachtenswerthen Berliner Dissertation vom Jahre 1881 „Beiträge zur physiologischen Wirkung des Aufenthalts in comprimirter Luft" an der Hand von Messungen des intrapleuralen Druckes bei Kaninchen nach der von Donders angegebenen Methode feststellen können, dass die Athmungsgrösse in comprimirter Luft wächøt. Leider hat er diese Untersuchungen nicht auch auf die Beeinflussung derselben durch verdünnte Luft ausgedehnt. Im Einklang mit diesen Untersuchungen stehen die Angaben, dass mittelst Percussion in verdichteter Luft ein tieferer Stand des Zwerchfells und eine verkleinerte Herzdämpfung nachgewiesen werden kann. Des ferneren wurde v. Vivenot beobachtet und nach diesem von vielen Forschern bestätigt, dass unter dem Einflusse comprimirter Luft die Athmung langsamer und tiefer werde, während dieselbe in verdünnter Luft Anfangs oberflächlicher und schneller, späterhin tiefer und forcirter werde. Ich selbst habe in zwei Arbeiten versucht, die Frage hinsichtlich des Effektes verdichteter und verdünnter Luft auf die Mechanik der Respiration zu ergänzen, einmal $^{3}$ ) an der Hand von Versuchen über den intratrachealen

1) Beiträge zur physiol. Wirkung der comprimirten Luft. Inaug.-Diss. Berlin 1877.

2) Ueber den Einfluss der verdünnten Luft auf den menschlichen Organismus. Inaug.-Diss, Berlin 1877.

3) Dieses Archiv. Bd. 130. Heft 2. S. 297. 
Druck beim Menschen und dann ${ }^{1}$ ) bei Gelegenheit von plethysmographischen Untersuchungen der Athembewegungen des Menschen.

Versuche über den intrapleuralen Druck bei Kaninchen, mit denen ich beschäftigt war, liessen es mir wünschenswerth erscheinen, Experimente anzustellen über die Einwirkung verdichteter und verdünnter Luft auf den Druck innerhalb der Pleurahöhle.

Wenn ich von einigen misslungenen Versuchen abstrahire, habe ich über sechs Versuchsreiben zu referiren, welche zur Entscheidung der aufgeworfenen Frage beitragen sollen.

A. Einfluss comprimirter Luft.

Die drei hierher gehörigen Versuche wurden am 15. December 1894, am 5. Januar und 19. Januar 1895 ausgeführt. Das Ergebniss derselben ist in den folgenden drei Tabellen niedergelegt, welche einiger Erläuterung bedürfen. Die in den Tabellen mitgetheilten Zahlen sind Mittelzahlen, berechnet aus 14 Einzelmessungen. Aus den Curven des Glycerinmanometers habe ich die intrapleuralen Druckwerthe in Quecksilberdruck umgerechnet und in den Tabellen nur diese aufgenommen. Der vierten Columne unserer Tabellen, welche über die Respirationsfrequenz Aufschluss geben, als Zeiteinheit zu Grunde gelegt habe ich die Zeit, welche erforderlich war, damit unser Papierstreifen $10 \mathrm{~cm}$ von der Kymographiontrommel entrollt wurde. Die jedesmalige Curvenzeichnung nahm ich vor bei: 1) Atmosphärendruck, 2) $1 \frac{1}{4}$ Atmosphärendruck, 3) $1 \frac{1}{2}$ Atmosphären, 4) nochmals $1 \frac{1}{2}$ Atmosphären, kurz bevor wir mit der Luftverdichtung nachliessen, 5) $1 \frac{1}{4}$ Atmosphären und 6) Atmosphärendruck. Die Zeiten, in denen gezeichnet warde, und die Temperaturen, welche während der Curvenaufnahmen in der pneumatischen Kammer herrschten, sind aus den Tabellen ersichtlich.

Ich darf mich wohl begnügen, den ersten Versuch bei comprimirter Luft etwas eingehender zu besprechen; das Resultat der beiden anderen dürfte dann wohl aus den mitgetheilten Tabellen von selbst verständlich sein.

1) Dieses Archiv. Bd. 132. Heft 3. S. 417. 
Während wir bei unserem Versuchsthier im Beginn der Beobachtung auf der Höhe der Inspiration einen intrapleuralen Druck von $-3,21 \mathrm{~mm} \mathrm{Hg}$ (sämmtliche Zahlen sind Mittelzahlen aus je 14 Einzelmessungen) bei Atmosphärendruck fanden, stieg derselbe bei $1 \frac{1}{4}$ Atmosphären auf $-3,88$, bei $1 \frac{1}{2}$ Atmosphären auf $-4,94 \mathrm{~mm} \mathrm{Hg}$. Wir liessen das Thier eine halbe Stunde unter diesem Drucke und erhielten am Ende dieser Zeit - $5 \mathrm{~mm} \mathrm{Hg}$. Als wir dann mit der Luftcompression in der Glocke nachliessen, sank der inspiratorische intrapleurale Druck in promptester. Weise conform mit der Druckverminderung. Bei $1 \frac{1}{4}$ Atmosphären erreichte derselbe $-4,41 \mathrm{~mm} \mathrm{Hg}$ und bei Atmosphärendruck $-3,22 \mathrm{~mm} \mathrm{Hg}$, fast genau den Anfangsdruck.

Betrachten wir jetzt den exspiratorischen, intrapleuralen Druck (siehe Columne 2 der Tabelle). Bei Beginn unseres Versuches betrug derselbe als Mittel aus 14 Einzelmessungen $-1,43 \mathrm{~mm} \mathrm{Hg}$. Er wuchs bei $1 \frac{1}{4}$ Atmosphären auf $-2,08$, bei $1 \frac{1}{2}$ Atmosphären auf $-3,73 \mathrm{~mm} \mathrm{Hg}$. Nach Ablauf einer halben Stunde bei dem gleichen Druck bekamen wir $-3,89 \mathrm{~mm} \mathrm{Hg}$. Hierauf sank der exspiratorische, intrapleurale Druck beim Nachlassen der Luftverdichtung und zwar bei $1 \frac{1}{4}$ Atmosphären auf $-2,18 \mathrm{~mm} \mathrm{Hg}$, um beim Uebergang in Atmosphärendruck $-1,57 \mathrm{~mm} \mathrm{Hg}$ zu betragen, also noch um ein Weniges mebr, als der exspiratorische Druck, von dem wir ausgingen.

Gehen wir nun die dritte Columne unserer Tabelle durch, welche den Einfluss comprimirter Luft auf die Tiefe der Athmung angiebt, so werden wir hier bei unseren drei Versuchsreihen keine Constanz finden. Nach den schon citirten Untersuchungen v. Vivenot's hätten wir ein Tieferwerden der Athmung und eine Verlangsamung derselbe erwarten müssen; beides trifft in unseren Experimenten, wenigstens nicht mit jener Sicherheit zu, wie es von v. Vivenot behauptet wird. -

Columne vier der Tabellen giebt Aufschluss über Veränderungen der Respirationsfrequenz bei unseren Versuchen. Während wir in unserem ersten Experimente mit verdichteter Luft in der That eine Verlangsamung der Respiration (s. Tabelle) haben constatiren können, konnten wir dies bei Versuch 2 und 3 nicht in gleicher Weise bestätigen.

Die letzte Columne unserer Tabellen zeigt den Blatdruck 
des Kaninchens an, gemessen in einer Carotis. Auch hier sind Mittelzahlen aus je 14 Messungen angegeben, und zwar wurde die Messung des Blutdruckes jedesmal auf der Höhe der Exspiration des Kaninchens ausgeführt. Auch bei den Ergebnissen dieser Untersuchungen können wir keine Regelmässigkeit erkennen; bald scheint der Blutdruck unter der Einwirkung der Luftcompression in der pneumatischen Kammer zu wachsen, bald wieder zu fallen. Im Allgemeinen verhält er sich derart, dass er Anfangs bei der Luftverdichtung zunimmt, um bei $1 \frac{1}{2}$ Atmosphären bald mehr, bald weniger abzunehmen. Wurde mit der Luftcompression nachgelassen, so hob sich der Blutdruck bei 1 $\frac{1}{4}$ Atmosphären um etwas und sank beim Uebergang in Atmosphärendruck sogar unter den Blutdruck, von dem wir ausgegangen waren. In Tabelle III finden wir in der letzten Columne nur 2 Zahlen angegeben, die übrigen fehlen, da hier in der Arterie Gerinnungen eingetreten waren, welche die weitere Beobachtung des Blutdruckes störten. Im Ganzen und Grossen stimmen die Ergebnisse unserer Untersuchungen hinsichtlich der Einwirkung comprimirter Luft auf den Blutdruck mit den Resultaten Jacobson's und Lazarus') in erfreulicher Weise überein.

Tabolle I.

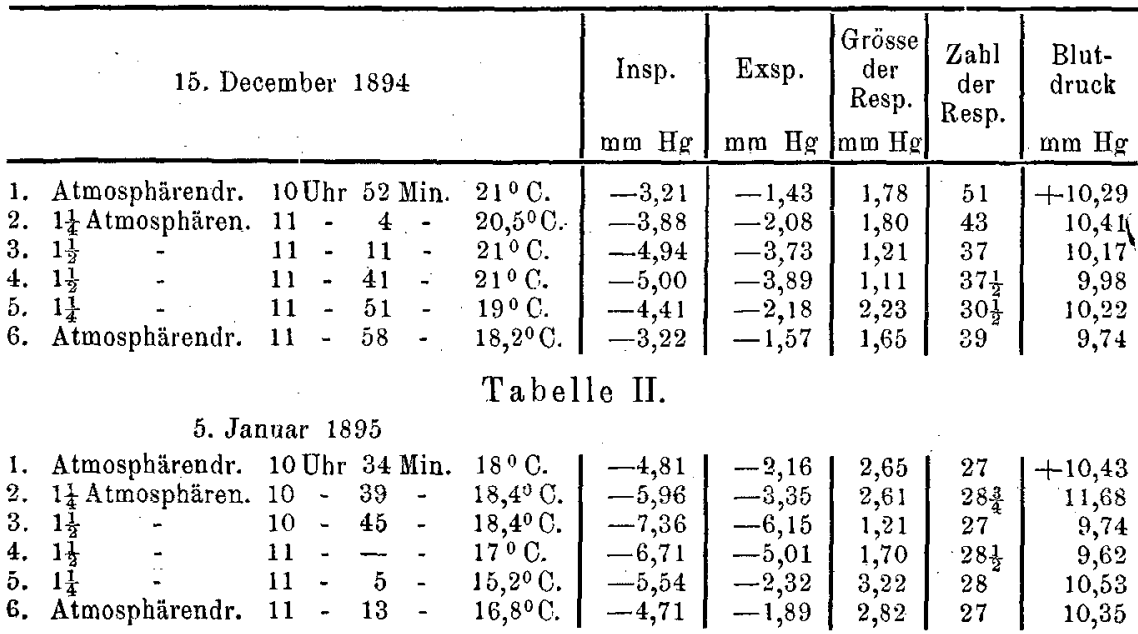

1) Centralbl. f. d. med. Wissensch. 1877. No.51. S. 929. 
Tabelle III.

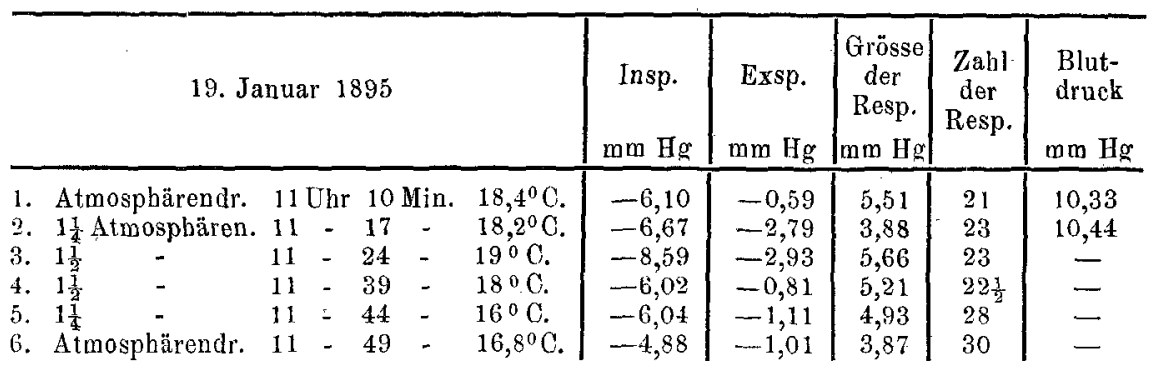

Das einzige Regelmässige, was wir bei unseren Experimenten haben also feststellen können, und was ja auch der eigentliche Gegenstand unserer Untersuchungen war, ist etwas Folgendes:

Der negative, intrapleurale Druck wächst beim Kaninchen mit zunehmender Compression der amgebenden Atmosphäre und sinkt wiederum mit $\mathrm{Nach}$ lassen der Luftuerdichtung.

Wächst der negative, intrapleurale Druck, so muss das Zwerchfell dementsprechend tiefer treten, wie wir im Beginne dieser Abhandlung näher aus einander gesetzt haben. Unter diesen Verhältnissen wird die Lunge stärker entfaltet und befindet sich mehr in Inspirationsstellung. Die Beeinflussung der Respiration unter $1 \frac{1}{2}$ Atmosphärendruck ist bereits eine sehr beträchtliche, der intrapleurale Druck ist um ein sehr Bedeutendes gewachsen.

Wir haben diese Versuche nur an Kaninchen ausgeführt. Aus diesen Experimenten werden wir wohl mit Recht unter Beobachtung von einiger Vorsicht auch Schlüsse auf die Beeinflussung des intrapleuralen Druckes anderer Thiergattungen und vielleicht auch des Menschen unter der Einwirkung comprimirter Luft ziehen dürfen. Vielleicht dürfen wir sogar voraussetzen, dass bei fleischfressenden Thieren und auch beim Menschen der Effekt ein noch hochgradigerer sein muss, als beim Kaninchen, das bekanntlich keine oder doch nur sehr wenig Darmgase besitzt, Bei allen Thierspecies mit Darmgasen wird eben jener bedeutungsvolle Factor noch hinzukommen müssen, welcher, wie Anfangs aus einander gesetzt wurde, bei der Deutung der Wirkung der Luftcompression eine so wichtige 
Rolle spielt. Werden die Darmgase in verdichteter Luft com. primirt, und nehmen sie daher dann ein kleineres Volum ein, so wird dieser Volumsverkleinerung entsprechend das Zwerchfell tiefer treten können. Tritt diese Wirkung hinzu, so werden wir voraussetzen dürfen, dass die Beeinflussung des intrapleuralen Druckes bei Thieren mit Darmgasen noch augenfälliger sein muss, als dies schon bei solchen ohne Darmgase der Fall war.

\section{B. Einfluss verdünnter Luft.}

Die Versuche über die Beeinflussung des intrapleuralen Druckes durch verdünnte Luft am 22. December 1894, 12. Januar und 2. Februar 1895 wurden in ganz der analogen Weise ausgeführt, wie die eben besprochenen mit comprimirter Luft. Wir gingen hierbei mit der Luftverdünnung bis auf $\frac{1}{2}$ Atmosphäre herunter, eine Luftverdünnung, in welcher ich selbst mich noch eben ohne all' $z \mathfrak{u}$ grosse Unannehmlichkeiten aufhalten kann. Die Kaninchen selbst athmen unter diesem Luftdruck, wenn er wenigstens, wie in unseren Versuchen, ziemlich brüsk in mässig kurzer Zeit hergestellt wird, in einer Weise angestrengt und beschleunigt unter Hinzuziehung sämmtlicher auxiliarer Respirationsmuskeln, dass man eine bedeutende Dyspnoe der Versuchsthiere bei $\frac{1}{2}$ Atmosphärendruck wohl kaum wird bestreiten können. Wenn man diesen dyspnoischen Zustand des Thieres auch nur einmal trotz Körperruhe unter diesen äusseren Verhältnissen mit eigenen Augen gesehen hat, so wird man wohl kaum jenen Stimmen beipflichten können, welche noch immer behaupten, dass der respiratorische Quotient bei einer derartigen Luftverdünnung unverändert bleibt. Wenn ein Kaninchen schon bei völliger Körperruhe unter $\frac{1}{2}$ Atmosphäre derart dyspnoisch ist, um wie viel mehr wird diese Dyspnoe sich steigern müssen, wenn es eine gewisse Arbeit noch za leisten hätte. Beim Menschen finden wir ähnliche, augenfällige Veränderungen der Athmung. in $\frac{1}{2}$ Atmosphäre, bei manchen Individuen schon, bevor wir bis auf $\frac{1}{2}$ Atmosphäre heruntergegangen sind, bei anderen vielleicht etwas später, so dass es schwer, um nicht zu sagen unverständlich erscheint, wie dabei der respiratorische Quotient unbeeinflusst bleiben könnte. Wenn die Respiration dyspnoisch und die sichtbaren Schleimhäute livide und 
cyanotisch werden, so kann der respiratorische Quotient doch unmöglich normal bleiben, wie dies von anderer Seite ${ }^{1}$ ) behauptet worden ist. Dann war eben der Organismus nicht mehr in der Lage durch Zuhülfenahme der Reservekräfte einen Ausgleich herbeizuführen. Ich kenne sogar Menschen, welche sich nur schwer wieder dazu entschliessen würden, sich in der pneumatischen Kammer die Luft auf $\frac{1}{2}$ Atmosphäre verdünnen zu lassen, welche noch Tage lang nachher recht unangenehme Nachwehen zu verspüren hatten. Und dieses alles trifft schon bei absoluter Körperruhe zu, um wie viel mehr erst bei Körperarbeit; da dürfte die zur Verfügung stehende Reservekraft noch viel eher versagen.

Bei Besprechung der Beeinflussung des intrapleuralen Druckes durch verdünnte Luft möchte ich unseren zweiten Versuch vom 12. Januar 1895 durchgehen; das Ergebniss der beiden übrigen dürfte aus den beigegebenen Tabellen dann von selbst verständlich sein.

Der intrapleurale Druck betrug bei unserem Kaninchen auf der Höhe der Inspiration unter Atmosphärendruck $-4,22 \mathrm{~mm} \mathrm{Hg}$, exspiratorisch $-3,35$. Bei $\frac{3}{4}$ Atmosphären fanden wir als Mittel $-4,10$ für die Inspiration, für die Exspiration $-2,62 \mathrm{~mm} \mathrm{Hg}$, bei $\frac{1}{2}$ Atmosphäre $-3,45$ und $-1,92 \mathrm{~mm} \mathrm{Hg}$. Bei der Luftverdünnung hatte sich also die Athmung des Thieres derart verändert, dass der negative intrapleurale Druck derselben sowohl auf der Höhe der Inspiration, als auch auf der Höhe der Exspiration kleiner wurde, und zwar bei $\frac{3}{4}$ Atmosphären kleiner als bei Atmosphärendruck und bei $\frac{1}{2}$ Atmosphäre noch kleiner als bei $\frac{3}{4}$ Atmosphäre. Die Lunge hatte also mehr eine exspiratorische Lungenstellung eingenommen. Das $Z$ werchfell war dementsprechend mehr in die Höhe getreten. Nachdem wir einige Zeit bei $\frac{1}{2}$ Atmosphären ausgehalten hatten, bekamen wir für die Inspiration - 3,55, für die Exspiration - 2,12 $\mathrm{mm} \mathrm{Hg}$. Mit dem Nachlassen der Luftverdünnung war der intrapleurale Druck unseres Kaninchens bei $\frac{3}{4}$ Atmosphären $-4,37$ und $-3,23 \mathrm{~mm}$ $\mathrm{Hg}$, um beim Uebergang in Atmosphärendruck $-5,45$ und

1) A. Loewy, Ueber die Respiration und Circulation unter verdünnter und verdichteter, sauerstoffarmer und sauerstoffreicber Luft. Bonn 1894. Arch. f. d. ges. Phys. Bd. 58 . 
$-3,91 \mathrm{~mm} \mathrm{Hg} \mathrm{zu}$ erreichen. Mit dem Nachlassen der Luftverdünnung wurde der intrapleurale Druck wieder grösser, entfaltete sich die Lunge wieder mehr und trat das Diaphragma wieder tiefer.

Hinsichtlich der Tiefe der Respirationen unter dem Einflusse der verdünnten Luft konnten wir, wie die dritte Columne unserer Tabellen ausweist, keine genügende Constanz eruiren; es scheint im Allgemeinen so zu sein, dass die Kaninchen bei $\frac{3}{4}$ Atmosphären tiefer athmen als unter Atmosphärendruck. Bei $\frac{1}{2}$ Atmosphäre wurde die Athmung zuweilen oberflächlicher, zuweilen wohl auch tiefer.

Was die Frequenz der Athmung betrifft, so konnten wir bestätigen, dass die Zahl der Athemzüge mit dem Grade der Luftverdünnung zunimmt und andererseits mit Nachlassen der Luftverdünnung wieder abnimmt, siehe Columne 4 der folgenden Tabellen.

Schliesslich möchte ich noch mit einigen wenigen Worten auf die Beeinflussung des Blutdruckes unter Luftverdünnung (s. Columne 5 der Tabellen) zurückkommen. Im Allgemeinen können wir nach unseren Versuchen behaupten, dass der Blutdruck (gemessen in der Carotis) mit zunehmender Luftverdünnung steigt und beim Nachlassen derselben fällt. Leider sind die von uns erhaltenen Resultate nicht mit jenen von Lazarus und Schirmunski ${ }^{1}$ ) in Einklang zu bringen, welche in ihren Experimenten gefunden haben, dass der Blutdruck bei einer Verdünnung um etwa $\frac{1}{2}$ Atmosphäre eine Verminderung erfährt, mit dem Wiederansteigen des Luftdruckes wieder zunimmt, ohne jedoch zu der Höhe, welche er bei Beginn des Versuches inne hatte, zurückzukehren. Fränkel und Geppert²) haben andererseits nach ihren Experimenten behauptet, dass „der arterielle Blutdruck unter dem Einfluss des verminderten barometrischen Drucks eine erhebliche Aenderung nicht erfährt“. Es dürfte nach alledem wünschenswerth sein, dass Blutdruckversuche in verdünnter Luft noch in grösserer Zahl angestellt werden, um zu einheitlichen Resultaten zu gelangen.

1) Zeitschr. f. klin. Med. Bd. VII. 1884. S. 299.

2) Ueber die Wirkungen der verdünnten Luft auf den Organismus. Berlin 1883. S. 72 . 
Tabelle I.

\begin{tabular}{lll|l|l|c|c|c}
\hline 22. December 1894 & & Insp. & Exsp. & $\begin{array}{c}\text { Grösse } \\
\text { der } \\
\text { Resp. } \\
\text { mm Hg }\end{array}$ & $\begin{array}{c}\text { Zahl } \\
\text { der } \\
\text { Resp. }\end{array}$ & $\begin{array}{c}\text { Blut- } \\
\text { druck }\end{array}$ \\
& & & & \\
mm Hg
\end{tabular}

Tabelle II.

12. Januar 1895

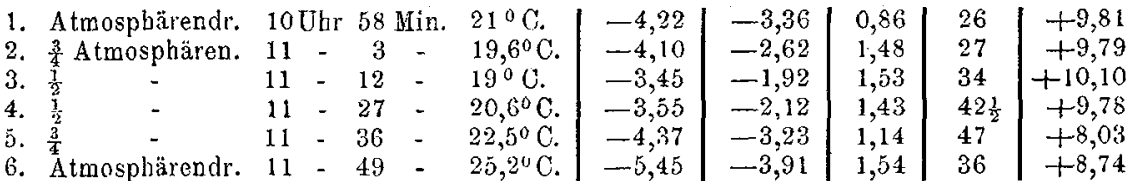

Tabelle III.

2. Februar 1895

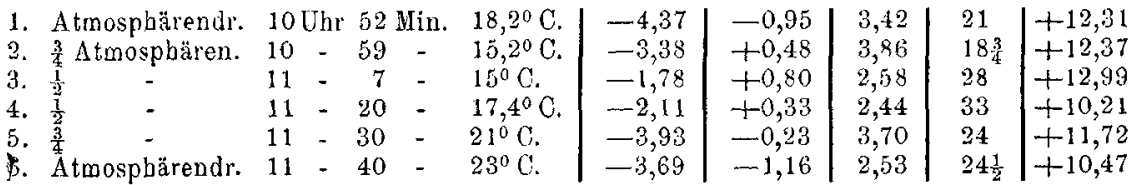

Constant haben wir also bei unseren Versuchen in verdünnter Luft Folgendes feststellen können:

Der negative, intrapleurale Drack sinkt beim Kaninchen mit zunehmender Luftverdünnung und wächst wiederum mit Nachlassen der Luftverdünnung.

Sinkt der negative, intrapleurale Druck, so muss das $Z$ werchfell dementsprechend hinaufsteigen. Unter diesen Bedingungen ist die Lunge weniger stark entfaltet und befindet sich mehr in Exspirationsstelluug. Die Beeinflussung der Athmung unter $\frac{1}{2}$ Atmosphärendruck ist eine sehr bedeutende, der intrapleurale Druck ist sehr nennenswerth gesunken.

Bei Thieren mit Darmgasen werden wir wohl auch bei Luftverdünnung einen noch grösseren Effekt auf den intrapleuralen Druck und die Athemmechanik überhaupt voraussetzen dürfen, indem hier ein wichtiges Moment noch hinzukommt. Die Darmgase werden nehmlich in verdünnter Luft ein grösseres 
Volum einnehmen müssen und so ihrerseits das $Z$ werchfell noch weiter in die Höhe drängen, wobei der intrapleurale Druck noch weiter wird sinken müssen.

Wenn auch die verdünnte Luft im Allgemeinen nicht zu Heilzwecken benützt wird, und sonst wenig Gelegenheit vorhanden ist, sich in so brüsker Weise, wie wir es ausgeführt haben, einer bedeutenden Luftverdünnung zu unterziehen (Ballonfahrten gehören doch noch zu den Seltenheiten), so liegt die praktische Bedeutung derartiger Untersuchungen in dem Beitrag zur Lösung der Frage, welche Fränkel, Geppert, von Liebig, Lazarus u. A. in ihren Arbeiten auch schon gestreift haben, dass der Aufenthalt im Hochgebirge und namentlich die modernen Reisen (Hochgebirgsbahnen) in dasselbe nicht unterschiedslos als völlig unschuldig in ihrer Einwirkung auf den Organismus betrachtet werden können.

Herrn San.-Rath Dr. Lazarus spreche ich an dieser Stelle für die Anregung und das lebhafte Interesse, das derselbe dieser Arbeit wiederum entgegengebracht hat, meinen verbindlichsten Dank aus. 